\title{
Absence of the Gribov ambiguity in a special algebraic gauge
}

\author{
Haresh Raval ${ }^{\mathrm{a}}$ \\ Department of Physics, Indian Institute of Technology Bombay, \\ Mumbai-400076, India
}

\begin{abstract}
Résumé. The Gribov ambiguity exists in various gauges except algebraic gauges. However in general, algebraic gauges are not Lorentz invariant, which is their fundamental flaw. Here we discuss a quadratic gauge fixing, which is Lorentz invariant. We show that nontrivial copies can not occur in this gauge. We then provide an example of spherically symmetric gauge field configuration and prove that with a proper boundary condition on the configuration, this gauge removes the ambiguity on a compact manifold $\mathbb{S}^{3}$.
\end{abstract}

\section{Introduction}

Defining the path integral in gauge theories has a major issue of infinite redundant functional integrations. The fact that the Yang-Mills action is invariant under the gauge transformation is the cause of the issue. The issue is addressed by invoking a gauge condition such as the Landau gauge $\partial^{\mu} A_{\mu}=f$. However, it is shown in ref. [1] that even after the Landau gauge fixing, there still exist a finite number of equivalent configurations, which contribute to the measure of the path integral. This implies that the Landau gauge does not uniquely choose a configuration, the problem known as the Gribov ambiguity. We need only inequivalent configurations in the measure in order to properly quantize the theory. The inequivalent configurations can be extracted out by restricting the space of integration to the fundamental modular region $C^{0}$, where the faddeev-poppov operator has positive eigenvalues [1]. However, the region $C^{0}$ still contains Gribov copies [1]. The restriction on the space of integration is achieved by adding suitable terms to the effective action $S_{\text {eff }}$ resulting from the Landau gauge fixing $[2,3]$. This modified action is known as Gribov-Zwanziger action. The same ambiguity is shown to exist in all the covariant gauges [4].

An essential reason why some gauges have the ambiguity is the differential operator involved in the gauge. Algebraic gauges are ambiguity free since they do not have a differential operator, but they have one disadvantage. In general, they violate the Lorentz invariance, which is a basic requirement for any theory. Whereas, the gauge under consideration in this paper is Lorentz invariant. Alternative formulations addressing the Gribov ambiguity are suggested in ref. [5, 6]. The former ref. particularly is an approach using Lorentz invariant algebraic gauge conditions.

The contents of this paper are arranged as follows : in the next section, we discuss a particular quadratic gauge and its consequences at infra-red scale. In sec. 3, we show that non trivial configurations can not occur in the present gauge. In sec. 4, we examine a case of the spherically symmetric gauge configuration. We prove that when a proper boundary condition on the gauge configuration at $\infty$

a e-mail: haresh@phy.iitb.ac.in 
is taken into account, the quadratic gauge uniquely chooses the configuration on a compact manifold $\mathbb{S}^{3}$.

\section{A quadratic gauge and Effective Lagrangian}

There have been studies using quadratic gauges in several contexts. A few of the references are [712]. Here we consider a particular quadratic gauge introduced in the ref. [13] in the context of infra-red phenomena in QCD.

$$
H^{a}\left[A^{\mu}(x)\right]=A_{\mu}^{a}(x) A^{\mu a}(x)=f^{a}(x) ; \text { for each } a
$$

where $f^{a}(x)$ is an arbitrary function of $x$. This gauge condition results in the effective Lagrangian of the form [13]

$$
\begin{aligned}
\mathcal{L}_{e f f} & =\mathcal{L}_{Y M}+\mathcal{L}_{G F}+\mathcal{L}_{\text {ghost }} \\
& =-\frac{1}{4} F_{\mu \nu}^{a} F^{\mu v a}-\frac{1}{2 \zeta}\left(A_{\mu}^{a} A^{\mu a}\right)^{2}-\overline{c^{a}} A^{\mu a}\left(D_{\mu} c\right)^{a}
\end{aligned}
$$

where the first term is Yang-Mills Lagrangian with $F_{\mu \nu}^{a}(x)=\partial_{\mu} A_{\nu}^{a}(x)-\partial_{\nu} A_{\mu}^{a}(x)-g f^{a b c} A_{\mu}^{b}(x) A_{\nu}^{c}(x)$, second and third terms are gauge fixing and ghost Lagrangian respectively and $\left(D_{\mu} c\right)^{a}=\partial_{\mu} c^{a}-$ $g f^{a b c} A_{\mu}^{b} c^{c}$.

The ghost Lagrangian contains a term $g f^{a b c} \bar{c}^{a} c^{c} A^{\mu a} A_{\mu}^{b}$. In the ghost condensed phase as shown in ref. [13], the term gives two strong signatures of the color confinement [13] 1. Abelian dominance and 2. A pole of the off-diagonal gluon propagator is on imaginary $p^{2}$ axis. The propagator not having poles on a real $p^{2}$ axis is a sufficient condition for the confinement [14].

In this paper, we aim to address another crucial non-perturbative phenomenon, the Gribov ambiguity. Thus, the purpose of this work with the results described above is also to justify the motivation and suitability of a choice of the quadratic gauge for the non-perturbative problems in QCD.

\section{Absence of Gribov copies in the quadratic gauge}

We now prove that non trivial Gribov copies do not exist in the present gauge due to its non linear nature. Under an infinitesimal gauge transformation $U=1+\alpha$, the quadratic gauge for the gauge transformed field is given by

$$
\begin{aligned}
\tilde{A}_{\mu}^{a} \tilde{A}^{\mu a} & =\left(A_{\mu}^{a}+\left(D_{\mu} \alpha\right)^{a}\right)\left(A^{\mu a}+\left(D^{\mu} \alpha\right)^{a}\right) \\
& =A_{\mu}^{a} A^{\mu a}+2 A^{\mu a}\left(D_{\mu} \alpha\right)^{a}+\left(D_{\mu} \alpha\right)^{a}\left(D^{\mu} \alpha\right)^{a}
\end{aligned}
$$

Hence, the gauge equivalence $\tilde{A}_{\mu}^{a} \tilde{A}^{\mu a}=A_{\mu}^{a} A^{\mu a}$ yields

$$
2 A^{\mu a}\left(D_{\mu} \alpha\right)^{a}+\left(D_{\mu} \alpha\right)^{a}\left(D^{\mu} \alpha\right)^{a}=0
$$

Therefore, $\left(D_{\mu} \alpha\right)^{a}=0 \Rightarrow \tilde{A}_{\mu}^{a}=A_{\mu}^{a} \quad$ or $\quad\left(D_{\mu} \alpha\right)^{a}=-2 A_{\mu}^{a} \Rightarrow \tilde{A}_{\mu}^{a}=-A_{\mu}^{a}$

So in neither case any new configuration is present. For the finite transformation also the same result is true. A finite transformation of the gauge field is given by

$$
\tilde{A}_{\mu}=A_{\mu}+i\left(D_{\mu} U\right) U^{-1}
$$


where $D_{\mu} U=\partial_{\mu} U+i\left[A_{\mu}, U\right]$. Now, it is easy to see that solving a gauge equivalence $\tilde{A}_{\mu}^{a} \tilde{A}^{\mu a}=A_{\mu}^{a} A^{\mu a}$ for each $a$, amounts to solving a gauge equivalence $\tilde{A}^{\mu} \tilde{A}_{\mu}=A_{\mu} A^{\mu}$. Hence, we solve a latter equivalence which gives the following eq.

$$
\begin{aligned}
i A_{\mu}\left(D^{\mu} U\right) U^{-1}+i\left(D^{\mu} U\right) U^{-1} A_{\mu}+i^{2}\left(D^{\mu} U\right) U^{-1}\left(D_{\mu} U\right) U^{-1} & =0 \\
\left\{A_{\mu}+\frac{i}{2}\left(D_{\mu} U\right) U^{-1}, i\left(D^{\mu} U\right) U^{-1}\right\} & =0
\end{aligned}
$$

Therefore, $i\left(D_{\mu} U\right) U^{-1}=0 \Rightarrow \tilde{A}_{\mu}=A_{\mu} \quad$ or $\quad i\left(D_{\mu} U\right) U^{-1}=-2 A_{\mu} \Rightarrow \tilde{A}_{\mu}=-A_{\mu}$ Multiplying with the generator $T^{a}$ and then taking a trace on both sides in each case would yield a configuration which we would have got by solving a former gauge equivalence. Thus, we conclude that the gauge is Gribov ambiguity free.

\section{Spherically symmetric gauge field configuration and the quadratic gauge}

Here we demonstrate that the quadratic gauge uniquely picks up a spherically symmetric configuration on a compact manifold $\mathbb{S}^{3}$, when a proper boundary condition on the field is required to be satisfied. Compactification of a euclidean space $\mathbb{R}^{N}$ to a compact manifold $\mathbb{S}^{N}$ is achieved by the condition $U(\infty)=I$ [4]. Since the space in this example is $\mathbb{R}^{3}$, the condition would compactify it to $\mathbb{S}^{3}$. We begin by adopting a parameterization for a vector potential shown in ref. [1]

$$
A_{i}=f_{1}(r) \frac{\partial \hat{n}}{\partial x_{i}}+f_{2}(r) \hat{n} \frac{\partial \hat{n}}{\partial x_{i}}+f_{3}(r) \hat{n} n_{i}, \quad i=1,2,3
$$

Where $n_{i}=\frac{x_{i}}{r}, \quad r=\sqrt{\Sigma x_{i}^{2}}, \quad \hat{n}=i n_{j} \sigma_{j} \quad \sigma_{j}$ are Pauli matrices, $\hat{n}^{2}=-1$. For simplicity we choose $A_{0}=0$. Now, the spherically symmetric operator is given by

$$
U=\exp \left(\frac{\alpha(r)}{2} \hat{n}\right)=\cos \left(\frac{\alpha(r)}{2}\right)+\hat{n} \sin \left(\frac{\alpha(r)}{2}\right)
$$

Therefore, the compactification condition $U(\infty)=I$ implies $\alpha(\infty)=4 \pi n ; n$ is an integer. The gauge transformation $A_{\mu} \longrightarrow \tilde{A}_{\mu}=U A_{\mu} U^{-1}+U^{-1}\left(\partial_{\mu} U\right)$ results in transformations of $f_{1}, f_{2}$ and $f_{3}$ as follows

$$
\begin{aligned}
\tilde{f}_{1} & =f_{1} \cos \alpha+\left(f_{2}+\frac{1}{2}\right) \sin \alpha \\
\tilde{f}_{2}+\frac{1}{2} & =-f_{1} \sin \alpha+\left(f_{2}+\frac{1}{2}\right) \cos \alpha \\
\tilde{f}_{3} & =f_{3}+\frac{1}{2} \dot{\alpha}
\end{aligned}
$$

where overdot indicates differentiation with respect to $r$. Now, a th component of $A_{i}$ can be derived using following formula

$$
A_{i}^{a}=\frac{1}{2} \operatorname{Tr}\left(A_{i} \sigma_{a}\right)
$$

after some algebra, we get

$$
\begin{aligned}
& A_{1}^{1}=i\left[f_{1}\left(\frac{1}{r}-\frac{x_{1}^{2}}{r^{3}}\right)+f_{3} \frac{x_{1}^{2}}{r^{2}}\right] \\
& A_{2}^{1}=i\left[-f_{1} \frac{x_{1} x_{2}}{r^{3}}+f_{2} \frac{x_{3}}{r^{2}}+f_{3} \frac{x_{1} x_{2}}{r^{2}}\right] \\
& A_{3}^{1}=i\left[-f_{1} \frac{x_{1} x_{3}}{r^{3}}-f_{2} \frac{x_{2}}{r^{2}}+f_{3} \frac{x_{1} x_{3}}{r^{2}}\right]
\end{aligned}
$$




$$
\begin{aligned}
& A_{1}^{2}=i\left[-f_{1} \frac{x_{1} x_{2}}{r^{3}}-f_{2} \frac{x_{3}}{r^{2}}+f_{3} \frac{x_{1} x_{2}}{r^{2}}\right] \\
& A_{2}^{2}=i\left[f_{1}\left(\frac{1}{r}-\frac{x_{2}^{2}}{r^{3}}\right)+f_{3} \frac{x_{2}^{2}}{r^{2}}\right] \\
& A_{3}^{2}=i\left[-f_{1} \frac{x_{2} x_{3}}{r^{3}}+f_{2} \frac{x_{1}}{r^{2}}+f_{3} \frac{x_{2} x_{3}}{r^{2}}\right] \\
& A_{1}^{3}=i\left[-f_{1} \frac{x_{1} x_{3}}{r^{3}}+f_{2} \frac{x_{2}}{r^{2}}+f_{3} \frac{x_{1} x_{3}}{r^{2}}\right] \\
& A_{2}^{3}=i\left[-f_{1} \frac{x_{2} x_{3}}{r^{3}}-f_{2} \frac{x_{1}}{r^{2}}+f_{3} \frac{x_{2} x_{3}}{r^{2}}\right] \\
& A_{3}^{3}=i\left[f_{1}\left(\frac{1}{r}-\frac{x_{3}^{2}}{r^{3}}\right)+f_{3} \frac{x_{3}^{2}}{r^{2}}\right]
\end{aligned}
$$

We now impose a boundary condition on $A_{k}^{j}$ s. We require that

$$
A_{k}^{j} \rightarrow 0 \text { as } \frac{1}{r}, \text { as } r \rightarrow \infty
$$

From Eq.s (11), it is clear that this condition is achievable and the boundary condition on $f_{1}, f_{2}$ and $f_{3}$ can be easily interpreted, which is as following

$$
f_{1}, f_{2} \rightarrow \text { const. as } r \rightarrow \infty \text { and } f_{3} \rightarrow 0 \text { as fast as } \frac{1}{r} \text { as } r \rightarrow \infty
$$

Here we make one note. We want to address the ambiguity on $\mathbb{S}^{3}$, therefore a boundary condition on $f_{3}$ needs to be little stronger (faster than $\frac{1}{r}$ as $r \rightarrow \infty$ ) because of the eq. for copies (24) that we shall come across later in the section. Hence, we consider a stronger condition on $f_{3}$ only. We will use these boundary conditions to prove our claim. We first evaluate a condition

$$
A_{i}^{a} A^{i a}=A_{1}^{a} A^{1 a}+A_{2}^{a} A^{2 a}+A_{3}^{a} A^{3 a} ; \text { for each } a
$$

For example taking $a=1$, the gauge above takes the form

$$
\begin{aligned}
A_{i}^{1} A^{i 1} & =\left(A_{1}^{1}\right)^{2}+\left(A_{2}^{1}\right)^{2}+\left(A_{3}^{1}\right)^{2} \\
& =-\left[\frac{f_{1}^{2}}{r^{2}}\left(1-\frac{x_{1}^{2}}{r^{2}}\right)+\frac{f_{2}^{2}}{r^{2}}\left(1-\frac{x_{1}^{2}}{r^{2}}\right)+f_{3}^{2} \frac{x_{1}^{2}}{r^{2}}\right]
\end{aligned}
$$

In spherical polar coordinates, the condition can be written as

$$
=-\frac{1}{r^{2}}\left(f_{1}^{2}+f_{2}^{2}\right)+\sin ^{2} \theta \cos ^{2} \phi\left(\frac{1}{r^{2}}\left(f_{1}^{2}+f_{2}^{2}\right)-f_{3}^{2}\right)
$$

Hence,

$$
\tilde{A}_{i}^{1} \tilde{A}^{i 1}=-\frac{1}{r^{2}}\left(\tilde{f}_{1}^{2}+\tilde{f}_{2}^{2}\right)+\sin ^{2} \theta \cos ^{2} \phi\left(\frac{1}{r^{2}}\left(\tilde{f}_{1}^{2}+\tilde{f}_{2}^{2}\right)-\tilde{f}_{3}^{2}\right)
$$

For $\tilde{A}_{i}^{1} \tilde{A}^{i 1}=A_{i}^{1} A^{i 1}$ to happen

$$
\frac{1}{r^{2}}\left[\left(\tilde{f}_{1}^{2}+\tilde{f}_{2}^{2}\right)-\left(f_{1}^{2}+f_{2}^{2}\right)\right]+\sin ^{2} \theta \cos ^{2} \phi\left[\tilde{f}_{3}^{2}-f_{3}^{2}-\frac{1}{r^{2}}\left(\left(\tilde{f}_{1}^{2}+\tilde{f}_{2}^{2}\right)-\left(f_{1}^{2}+f_{2}^{2}\right)\right)\right]=0
$$


Since $\alpha$ is a function of $r$ only, the first term and the coefficient of $\sin ^{2} \theta \cos ^{2} \phi$ in a second term of Eq. (18) must individually vanish, giving us two different copy equations

$$
\begin{gathered}
\tilde{f}_{1}^{2}+\tilde{f}_{2}^{2}=f_{1}^{2}+f_{2}^{2} \Rightarrow f_{2}+\frac{1}{2}=-f_{1} \cot \frac{\alpha}{2} \\
\tilde{f}_{3}^{2}=f_{3}^{2} \Rightarrow f_{3} \dot{\alpha}+\frac{1}{4} \dot{\alpha}^{2}=0 \Rightarrow \quad \dot{\alpha}=0 \text { or } \dot{\alpha}=-4 f_{3}
\end{gathered}
$$

For a copy to exist, Eq. (19) has to be satisfied, with a parameter $\alpha$ in it satisfying either of two eq.s in Eq. (20). There are two choices to make since $f_{1}$ and $f_{2}$ are arbitrary functions.

1. $f_{2}+\frac{1}{2} \neq-f_{1} \cot \frac{\alpha}{2}$

2. $f_{2}+\frac{1}{2}=-f_{1} \cot \frac{\alpha}{2}$.

If

$$
f_{2}+\frac{1}{2} \neq-f_{1} \cot \frac{\alpha}{2}
$$

then it is clear that no copy exists for this choice.

However, if

$$
f_{2}+\frac{1}{2}=-f_{1} \cot \frac{\alpha}{2}
$$

then we encounter two copies corresponding to eq.s $\dot{\alpha}=0$ and $\dot{\alpha}=-4 f_{3}$. They are obtained by putting Eq. (22) in the transformation (9)

$$
\begin{aligned}
& \tilde{f}_{1}=-f_{1} \\
& \tilde{f}_{2}=f_{2}
\end{aligned}
$$

Therefore for $\dot{\alpha}=0$ (putting $\dot{\alpha}=0$ back in transformation (9) ), we obtain

$$
\tilde{f}_{1}=-f_{1}, \quad \tilde{f}_{2}=f_{2}, \quad \tilde{f}_{3}=f_{3}
$$

which yields a copy

$$
\begin{aligned}
& \tilde{A}_{j}^{j}=A_{j}^{j}-2 i f_{1}\left(\frac{1}{r}-\frac{x_{j}^{2}}{r^{3}}\right) \\
& \tilde{A}_{k}^{j}=A_{k}^{j}+2 i f_{1} \frac{x_{j} x_{k}}{r^{3}}
\end{aligned}
$$

However, on a compact manifold $\mathbb{S}^{3}$ this copy no longer exists. Because $\dot{\alpha}=0 \Rightarrow \alpha=$ const. everywhere including infinity. Setting $\alpha(r)=\alpha(\infty)=4 \pi n$, for which the copy Eq. (22) implies $f_{2}=\infty$ everywhere giving a copy which is also $\infty$. We want finite copies of $A_{k}^{j}$ which is well behaved and finite at finite distances, which is not possible for $\dot{\alpha}=0$ on $\mathbb{S}^{3}$. Therefore, the Eq. (22) is not valid on $\mathbb{S}^{3}$, thus the copy vanishes on it.

Now, we are left with only one copy which corresponds to

$$
\dot{\alpha}=-4 f_{3} \Rightarrow \alpha=-4 \int f_{3} d r+\text { const } .
$$

Putting $\dot{\alpha}=-4 f_{3}$ back in transformation (9), we get

$$
\tilde{f}_{1}=-f_{1}, \quad \tilde{f}_{2}=f_{2}, \quad \tilde{f}_{3}=-f_{3}
$$


which yields a copy

$$
\begin{aligned}
\tilde{A}_{j}^{j} & =-A_{j}^{j} \\
\tilde{A}_{k}^{j} & =-A_{j}^{k}
\end{aligned}
$$

It can also be removed on $\mathbb{S}^{3}$. We recall boundary conditions (13). Since $f_{3} \rightarrow 0$ faster than $\frac{1}{r}$ as $r \rightarrow$ $\infty$, Eq. (24) $\Rightarrow \alpha(\infty)=$ const.. As for the previous copy, we set $\alpha(\infty)=4 \pi n$ for which Eq. (22) implies $f_{2} \rightarrow \infty$ as $r \rightarrow \infty$. Hence it is clear that on $\mathbb{S}^{3}$, Eq. (22) is an obstruction for the boundary condition on $f_{2}$ (Eq. (13)) to be satisfied therefore not valid. Therefore this copy does not exist on $\mathbb{S}^{3}$.

The result is true for stronger boundary conditions such as $\frac{1}{r^{2}}$ and $e^{-r}$. Similarly, it can be shown that the condition for other two components, $\tilde{A}_{i}^{2} \tilde{A}^{i 2}=A_{i}^{2} A^{i 2}$ and $\tilde{A}_{i}^{3} \tilde{A}^{i 3}=A_{i}^{3} A^{i 3}$, produce same two equations for copy.

Whereas for coulomb gauge, we have [2]

$$
\frac{\partial A_{i}}{\partial x_{i}}=\hat{n}\left(\dot{f}_{3}+\frac{2}{r} f_{3}-\frac{2}{r^{2}} f_{1}\right)
$$

Because Pauli matrices $\sigma_{a}$ are unit vectors in $2 \times 2$ matrix space, the condition

$$
\frac{\partial \tilde{A}_{i}^{a}}{\partial x_{i}}=\frac{\partial A_{i}^{a}}{\partial x_{i}}
$$

for all three components yields the equation

$$
\ddot{\alpha}+\frac{2}{r} \dot{\alpha}-\frac{4}{r^{2}}\left(\left(f_{2}+\frac{1}{2}\right) \sin \alpha+f_{1} \cos \alpha\right)=0
$$

This equation is known to be solvable and therefore the ambiguity exists.

\section{5 conclusion}

We discussed a particular quadratic gauge, which is a Lorentz invariant algebraic gauge and showed how the non linearity does not allow the ambiguity to exist. Later we worked out an example of spherically symmetric configuration in the quadratic gauge and proved that the configuration with a proper boundary condition does not have any copy on $\mathbb{S}^{3}$. Thus, through the work in this paper with the results of ref. [13], the choice of the quadratic gauge for infra-red phenomena is also justified.

\section{Acknowledgment}

Haresh is sincerely thankful to Professor Urjit A. Yajnik for useful comments on the subject.

\section{References}

[1] V. N. Gribov, Nucl. Phys. B139 1 (1978)

[2] D. Zwanziger Nucl. Phys. B323 513-544 (1989)

[3] D. Zwanziger, Nucl. Phys. B321 591-604 (1989)

[4] I. Singer, Comm. Math. Phys. 607 (1978)

[5] A. A. Slavnov, JHEP 08047 (2008)

[6] J. Serreau and M. Tissier, Phys. Lett. B 712 97-103 (2012) 
[7] Ken-ichi SHIZUYA, Nucl. Phys., B109 397-420 (1976)

[8] S. Weinberg, Phys. Lett. B 91 51-55 1980

[9] Ashok Das, Pramana 16 409-416 (1981)

[10] Ashok Das, M.A. NAMAZIE, Phys. Lett. B 99 463-466 (1981)

[11] S. D. Joglekar, Phys. Rev. D 104095 (1974)

[12] S. D. Joglekar and B. P. Mandal, Phys. Rev. D 51, 1919 (1995)

[13] Haresh Raval and Urjit A. Yajnik, Phys. Rev. D 91, 085028 (2015)

[14] C. D. Roberts, A. G. Williams, and G. Krein, Int. J. Mod.Phys. A 07, 5607 (1992) 